\title{
Reverberation: source of potential artifacts occurring during ultrasound-guided regional anesthesia
}

\author{
Theodosios Saranteas, MD · Andreas Karabinis, MD
}

Received: 20 October 2008/Revised: 15 November 2008/Accepted: 19 November 2008/Published online: 31 December 2008

(C) Canadian Anesthesiologists' Society 2008

\section{To the Editor,}

Image artifacts that emerge during diagnostic imaging include extraneous ultrasound signals appearing as structures that are not actually present or as structures that differ in size and/or shape from their actual appearance. ${ }^{1}$ Reverberations, an important source of artifacts in ultrasonography, are caused by the repeated back-and-forth reflections of an ultrasound wave between two strong specular reflectors. When the transducer reflects the strong echoes, they travel back to the original target. However, the transducer takes twice the time to detect this pulse than it does to detect the original pulse and, as a result, incorrectly places the target at twice the distance. Since this "second trip" duplicates the travel distance and the travel time, the targeted structure is imaged once at the correct distance and a second time at twice the distance from the transducer. As a result, an artifact is produced that appears as a duplication of the real structure. ${ }^{2}$

Cardiac anesthesiologists are familiar with reverberation artifacts. They recognize this phenomenon when they observe a double image of the heart (caused by reflection of the pericardium) while performing transesophageal echocardiography or transthoracic echocardiography. While most cardiac anesthesiologists are accustomed to such reverberatory effects, this manifestation may be less commonly recognized by anesthesiologists practicing regional anesthesia. Recently, while attaining supraclavicular

T. Saranteas, MD $(\bowtie)$

School of Medicine, University of Athens, Attikon Hospital,

Athens, Greece

e-mail: saranteas@ath.forthnet.gr

\author{
A. Karabinis, MD \\ General State Hospital of Athens, Athens, Greece
}

ultrasound identification of the brachial plexus, we observed the subclavian artery image in the transverse scan presenting as the source for a reverberatory artifact. The vessel was correctly imaged in the near field and falsely duplicated below (Fig. 1). More interestingly, the illusion of the subclavian artery was clearly visualized below the pleural line, creating a mirror-like image of the vessel within the lung. Immediate recognition of the artifact was instrumental to ensuring a successful block and an uneventful outcome.

In conclusion, ultrasound reverberations can potentially result in misinterpretation of the "real" image during

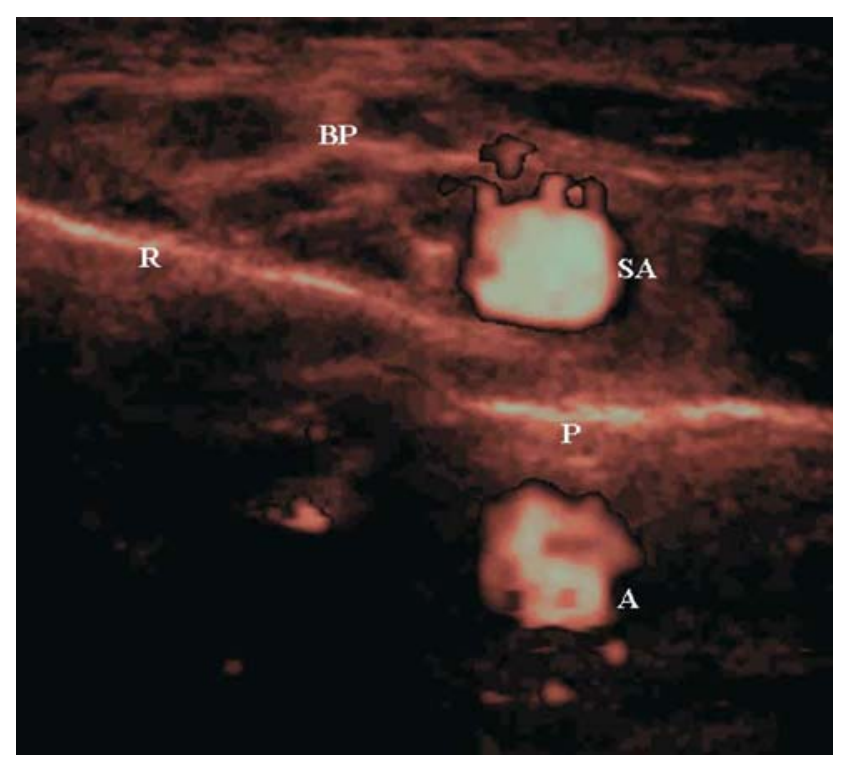

Fig. 1 A reverberatory image of the subclavian artery (SA). The vessel is visualized in the near field, together with the brachial plexus (BP). The duplicate reverberatory artifact (A) is observed within the lung, below the pleura $(\mathrm{P})$ and the first rib (R) 
ultrasound-guided regional anesthesia, as in this case, and can negatively influence patient outcome. For this reason, the education and training of specialists practicing ultrasound-guided regional anesthesia should include the recognition of ultrasound-related artifacts.

Conflicts of interest None declared.

\section{References}

1. Kremkau F, Taylor $K$. Artifacts in ultrasound imaging. J Ultrasound Med 1986; 5: 227-37.

2. Feigenbaum $H$. Physics and instrumentation. In: Feingenbaum $\mathrm{H}$, Armstrong WF, Ryan T editors. Feigenbaum's Echocardiography. Philadelphia: Lippincott Williams and Wilkins; 2005: 29-32. 